\title{
ON SECONDARY SPECTRA AND THE CONDITIONS UNDER WHICH THEY MAY BE PRODUCED.
}

By P. G. Nutring.

Since early in the development of spectroscopy ${ }^{a}$ it has been well known that some of the elementary gases electrically excited give two entirely different spectra. One of these, called by Plücker and Hittorf the primary spectrum, usually consists of a large number of broad lines well distributed over the whole visible and neighboring ultra violet spectrum. The secondary spectrum, on the other hand, consists usually of but few lines, and these very prominent. It is the spectrum obtained when capacity is connected in parallel with the tube. The two spectra are easily obtained with an ordinary Plücker tube having not too long nor too fine a capillary and containing nitrogen, sulphur, or iodine vapor at a pressure of from 2 to 10 millimeters. With a large condenser (at least one-twentieth microfarad) in parallel, such a tube shows a secondary spectrum; without the condenser it shows the primary spectrum if the current be not excessive. Some gases which exhibit secondary spectra also show anode and cathode glows having quite different spectra. The present investigation was undertaken to separate, in the Mendeleef system, those elements which show multiple spectra from those that do not, and to determine what conditions govern the production of secondary spectra.

Starting with the seventh group of the periodic system, it was found that chlorine, bromine and iodine, all of the group that could be worked with, gave multiple spectra. The effect is particularly striking with bromine and iodine. Likewise all of the sixth group available gave multiple spectra, namely oxygen, sulphur, selenium and tellurium. In the fifth group nitrogen, phosphorus and arsenic exhibit

$a$ Plücker and Hittorf: "On the Spectra of Ignited Gases and Vapors, with Special Regard to the Different Spectra of the Same Elementary Gaseous Substance." Phil. Trans., 155, pp. 1-29; 1865. 
multiple spectra while bismuth does not. With arsenic, however, the primary (anode) and secondary spectra have two prominent green lines in common. In the fourth group only the more metallic elements-tin and lead-could be used in a Plücker tube. The spectra of these was found not to change on connecting a condenser in shunt. In the third group aluminum, indium and thaliium; and in the second group magnesium, zinc, cadmium, and mercury give only line spectra and these are not essentially altered by capacity in parallel. Hydrogen excepted, the first group shows only single spectra. Without taking up the much-discussed ${ }^{a}$ question of the rôle of water vapor in gas conduction, from the point of view of multiple spectra the evidence is very strong that the fine-line spectrum of hydrogen is a true primary spectrum while the "four-line" is a true secondary. The latter is relatively enhanced under the same conditions that bring out the secondary spectra of other multiple-spectra elements. The primary and secondary spectra of other elements, notably chlorine and bromine, may be made to coexist in the same way, and the presence of water vapor certainly favors the preponderance of the secondary spectra of other elements as well as of hydrogen. Oxygen and sulphur are striking examples.

In the helium group there are wide differences. Helium itself shows but a single spectrum. Anode and cathode glows give identical spectra and both remain practically unchanged on the passage of the condenser discharge. Argon, however, shows at least four widely different spectra. The red primary (anode) and blue secondary spectra have been described by several observers. ${ }^{b}$ But the cathode glow shows quite a different spectrum from the anode (capillary) glow. With low dispersion it appears nearly continuous in the yellow, green and blue, while the strong red line and violet group of the anode glow are lacking. When the blue condenser discharge is produced in the capillary there is a buff-colored glow in the bulbs at either end. The spectrum of this glow shows the blue group lacking, but the yellow group of the red primary anode spectrum present. This difference between bulb and capillary spectra can hardly be regarded as other than a current density effect, since this is the chief, if not the only, difference in the excitation. As we should expect, the bulb spectrum with condenser is intermediate between the capillary spectrum with

$a$ Callendar: Nat. 56, p. 624; 1897. Trowbridge: Phil. Mag. [6], 2, p. 370; 1901. P. Lewis: Phil. Mag. [6], 3, p. 512; 1902. Parsons: Astroph. J., 18, p. 112; 1903.

${ }^{b}$ Trowbridge and Richards: Phil. Mag. [5], 43, p. 77; 1897. Kayser: Berlin Akad., 1896; Travers, Expl. Study of Gases, 1901, p. 312.

cBaly: Chem. News, 88, p. 26; 1903. 
condenser and the red capillary spectrum without condenser. Of neon, krypton, and zenon only the spectra of the two latter are said to be seriously affected by the addition of a condenser. ${ }^{c}$ No differences between anode and cathode capillary and bulb spectra appear to have been recorded.

In the accompanying Mendeléef table the elements showing multiple spectra are indicated by heavy-faced type. Elements showing distinct anode and cathode spectra are bracketed.

\begin{tabular}{|c|c|c|c|c|c|c|c|}
\hline 0 & I & II & III & IV & $\mathrm{V}$ & VI & VII \\
\hline $\begin{array}{l}\mathrm{He} \\
\mathrm{Ne}(\mathbf{A}) \\
-\quad \mathbf{K r} \\
-\quad \mathbf{X e}\end{array}$ & $\begin{array}{lr}\mathrm{H} & \mathrm{Li} \\
\mathrm{Na} & \mathrm{K} \\
\mathrm{Cu} & \mathrm{Rb} \\
\mathrm{Ag} & \mathrm{Cg} \\
\mathrm{Au} & \end{array}$ & $\begin{array}{ll}\mathrm{Mg} & \mathrm{Be} \\
\mathrm{Zn} & \mathrm{Ca} \\
\mathrm{Cd} & \mathrm{Sr} \\
\mathrm{Hg} & \mathrm{Ba}\end{array}$ & $\begin{array}{cc}\mathrm{Al} & \mathrm{B} \\
& \mathrm{Sc} \\
\mathrm{Ga} & \mathrm{Y} \\
\mathrm{In} & \mathrm{La} \\
\mathrm{Tl} & \end{array}$ & $\begin{array}{cc}\mathrm{Si} & \mathbf{C} \\
\mathrm{Ge} & \mathrm{Ti} \\
\mathrm{Sn} & \mathrm{Zr} \\
\mathrm{Pb} & \mathrm{Ce}\end{array}$ & $\begin{array}{lr}\mathbf{P} & (\mathbf{N}) \\
\mathrm{As} & \mathbf{V} \\
\mathrm{Sb} & \mathrm{Nb} \\
\mathrm{Bi} & \mathrm{Di}\end{array}$ & $\begin{array}{lr}\text { S } & (0) \\
\text { Se } & \text { Cr } \\
\text { Te } & \text { Mo } \\
- & -\end{array}$ & $\begin{array}{ll} & \text { F } \\
\text { Br } & - \\
\mathrm{I} & - \\
- & -\end{array}$ \\
\hline
\end{tabular}

Excepting then the anomalous helium group and hydrogen, we may say that in general, so far as observations go, the acid-forming elements have multiple spectra, while metallic elements do not.

Most metallic spectra are greatly altered by the addition of capacity, it is true, but the changes produced are by no means comparable with the change from primary to secondary, while metallic spectra, by every test known, are already secondary spectra-before adding capacity. They are series spectra, show the Zeeman effect and the displacement effect due to pressure, and the new lines brought in by added capacity are of the same (secondary) character. That so many metallic vapors exhibit banded absorption spectra would seem to indicate that metals may have primary spectra as well as nonmetals, but if so they are to be sought in the electrodeless discharge and cathode luminescence where the excitation is very feeble, rather than in the condenser discharge. Nor would the oxyhydrogen flame, banded spectra of the metals studied by Hartley and Ramage be comparable with primary spectra. In these spectra the lines of the series spectra simply become the heads of the bands in the flame spectra, while the lines of a true secondary spectrum appear to have no relation to the bands of the corresponding primary. Another notable distinction between the spectra of electro-negative and electro-positive elements is that the former, both primary and secondary, are remarkably invariant, while it is difficult to obtain the spectrum of a metal twice alike. 
Why should a condenser discharge produce a secondary spectrum? The oscillation frequency of a condenser is so much less than the frequency of collision of an electron in a gas at $1 \mathrm{~mm}$ pressure ${ }^{a}$ that an electron must collide a great number of times before the impelling force is reversed. Hence the oscillatory nature of the discharge per se could have little effect on the spectrum of a gas. On the other hand, the current density during discharge is thousands of times as great as during the steady flow of the same current. In testing other gases for the effect of excessive current density, specially designed Plücker tubes were used. One form had a very fine (thermometer) capillary and hollow cylindrical electrodes; another had a third bulb interposed in the middle of the capillary. In this way the current density could be increased a hundred or thousand times in parts of the tubes. Bromine, iodine, and hydrogen easily show the current density effect within the range of current that a common glass tube will carry. The primary spectrum changes continuously over into the secondary spectrum as the current is increased. Nitrogen and the sulphur group failed to show the secondary spectrum with the largest steady currents that glass tubes could carry. Argon is so sensitive to changes in current density that, in conjunction with a pocket spectroscope, it might be used as a milliammeter.

It was further undertaken to determine how much capacity was necessary to just produce the secondary spectrum in different gases, how this critical capacity varies with the spectral wave length, with the density of the gas, the amount of inductance and resistance in circuit, distance apart of electrodes, and sectional area of the dise charge. In this work photographic methods were employed throughout. Spectra obtained under varied conditions were photographed side by side on the same plate so that the minutest changes could be observed and followed. For this purpose a large model Fuess quartz spectrograph was used. This was provided with a large flint glass prism giving a spectrum about $15 \mathrm{~cm}$ long from 300 to $600 \mu \mu$. Ten or more spectra could be recorded on the same plate. A large glass condenser was used, composed of 20 plates well separated and provided with mercury cups, so that the capacity could be varied by a plate at a time. Current was supplied by transformers of 1,000, 2,000 and 5,000 volts, and by a set of generators giving 5,000 volts continuous current. For inductance, a Seibt tuning solenoid of 120 turns $20 \mathrm{~cm}$ in diameter was used. The greater part of the work was done with ordinary short, stout Plücker tubes made by Boehm, of Chicago. These had electrodes about $4 \mathrm{~cm}$ apart, and capillary por- 
tions $2 \mathrm{~mm}$ in diameter and $12 \mathrm{~mm}$ long. Some of the work was done with tubes without any central constriction. To keep the pressure of the inclosed gas more nearly constant, a half liter bulb was sealed to each tube while in use.

With a tube of air at $13 \mathrm{~mm}$ pressure spectra were photographed with capacities of $0.12,0.09,0.06,0.03$ microfarad and with no capacity in parallel. A sudden change from secondary to primary spectrum was found to occur at a capacity of about 0.05 microfarad, equivalent to that of about 14 one-gallon Leyden jars. Adding capacity indefinitely above 0.06 microfarad produced little, if any, effect, nor do secondary lines usually appear in the primary spectrum until the capacity is nearly 0.03 microfarad.

Critical capacity and wave length.-Drawing a line separating primary and secondary spectra on the photographic plate (see Pl. I) the ordinates of the curve represent roughly critical capacity, abscissas wave length. The curve drops off very steeply on the short wave length end in spite of the greater dispersion, indicating that for waves perhaps not shorter than $300 \mu \mu$ the critical capacity becomes infinite. Critical capacity expressed as a function of wave length appears to be of the form

$$
C=a e^{\frac{b}{\lambda-\lambda_{0}}}
$$

Approximate numerical results for air are given in the table:

\begin{tabular}{|c|c|c|c|}
\hline \multirow{2}{*}{ Pressure } & \multicolumn{3}{|c|}{ Wave length in $\mu \mu$. } \\
\hline & 350. & 450. & 550. \\
\hline & Microfarad. & Microfarad & Microfarad. \\
\hline $20 \mathrm{~mm} . . .$. & 0.07 & 0.03 & 0.02 \\
\hline $1 \mathrm{~mm} . . .$. & $>0.15$ & 0.06 & 0.03 \\
\hline
\end{tabular}

Critical capacity and pressure.-The critical capacity increases slightly as the pressure decreases down to about $2 \mathrm{~mm}$ air pressure, when it suddenly becomes infinite, i. e., no amount of capacity (without an external spark gap in series) will cause the secondary spectrum to appear. With spark gap, secondary spectra may be obtained at pressures of but a few tenths of a millimeter, nearly to the pressure at which cathode-ray fluorescence appears. This critical minimum pressure at which the disruptive discharge becomes possible is considerably 
lower for hydrogen (about $1 \mathrm{~mm}$ ), but depends on the condition of the surface of the electrodes and the presence of impurities. At high pressures the critical capacity continues to gradually decrease. With a spark in open air, either the primary or secondary nitrogen spectrum may be obtained according to the electrical conditions in circuit. Using a special tube the distance apart of whose electrodes could be varied to suit the potential ( 5,000 volts) employed, the critical capacity of air and of hydrogen was observed at various pressures up to atmospheric pressure. At atmospheric pressure it was found to be about one-fourth what it was at $10 \mathrm{~mm}$ pressure. The critical capacity appears to be roughly inversely proportional to the cube root of the pressure, or directly proportional to the mean distance apart of the molecules.

Critical capacity and nature of the gas.-Hydrogen, sulphur, nitrogen, oxygen, bromine, and iodine were tested and the critical capacity found to be practically the same for all for the same pressure and wave length. Critical capacity is more sharply marked in sulphur, nitrogen, and iodine. With hydrogen the lines of the secondary (four-line) spectrum invariably appear in the primary (many-line) spectrum, the capacity of the wires leading to the tube being a considerable factor in their dominance. All the substances show the same great increase in critical capacity for the very short wave lengths and decrease with increasing pressure.

Critical capacity appears to be nearly or quite independent of the voltage employed (up to 5,000 volts) and of the distance apart of the electrodes. Tests were made with the electrodes at various distances down to $3 \mathrm{~mm}$ apart. At much shorter distances the metallic lines from the electrodes become prominent at high pressure. Critical capacity increases with rise in temperature of the electrodes. No measurements were made, but care was taken during other tests to have the temperature of the electrodes fairly constant.

In the capillary of a Plücker tube the critical capacity is less than in the bulb or less than in a tube without a central constriction. This is confirmatory to a view expressed in a previous part of this paper that the production of a secondary spectrum was not so much the effect of capacity per se as of increased current density.

Critical capacity and inductance.-The effect of introducing inductance is always to relatively weaken the secondary and enhance the primary spectrum. But introducing a certain inductance is by no means equivalent in its effect to removing a definite corresponding capacity. Inductance was added in steps of 0.008 millihenry. The first inductance added, though very small, weakened the secondary 
spectrum very markedly and introduced primary lines, and this whether the capacity used was just above the critical capacity or five times that amount. Adding more and more inductance produces less and less effect. Apparently no amount of inductance, however great, will completely annul the effect of any capacity, however small. Capacity and inductance effects are shown graphically in the figure. Ordinates represent the change from primary to secondary spectra.

Critical capacity and resistance.-The effect of resistance is as pronounced as that of inductance in changing the secondary spectrum back to the primary. Even as little as $20 \mathrm{ohms}$ (non inductive) resistance brings in primary lines, while $100 \mathrm{ohms}$ gives a nearly pure primary. The resistance effect curve has very nearly the same form as the inductance effect curve, as shown in the figure. The effect of an external spark gap is always to favor the production of a secondary

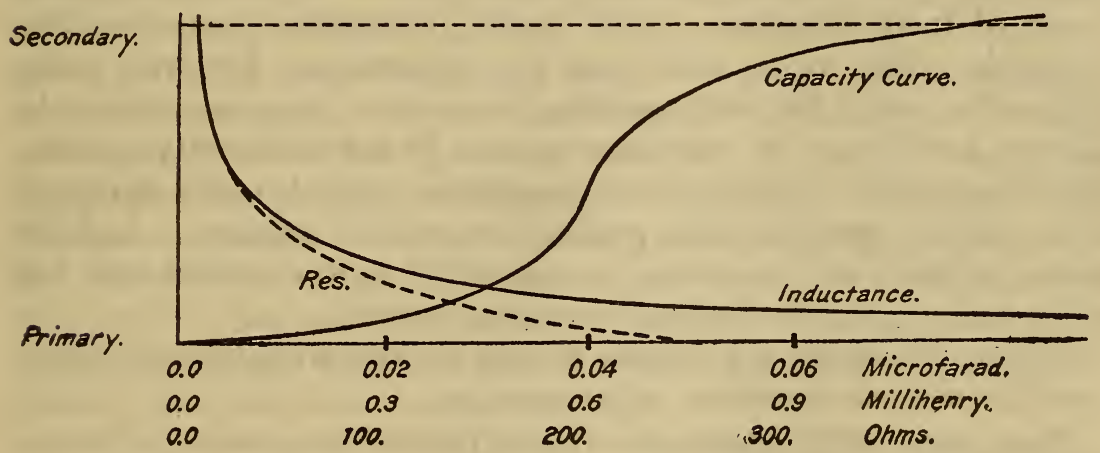

Fig. 1.

spectrum. Secondary spectra may be obtained with much less capacity and at much lower pressures with spark gap than without. But inductance appears to have the same effect with spark gap as without, while the ohmic resistance of the gap must be less than $10 \mathrm{ohms}$, judging by its effect on the discharge.

Critical capacity of mixtures.-Mixtures of hydrogen and nitrogen, sulphur and hydrogen, iodine and nitrogen, iodine and hydrogen, nitrogen and sulphur, hydrogen and oxygen, and mercury and nitrogen were tested, and each component was found to have its own critical capacity independently of the presence of the others. In a previous paper (The Spectra of Mixed Gases, this bulletin) it was shown that the relative intensities of two primary spectra (of a mixture of gases) depended on the relative atomic weight of the component gases, while the relative intensities of the secondary spectra depend only on the relative numbers of the different kinds of atoms present. For example, 
it is easy to prepare a tube of mixed hydrogen and nitrogen that shows a ("four-line" secondary) hydrogen spectrum with capacity but a nitrogen (primary) spectrum without.

To obtain a pure primary spectrum, then, it is necessary to avoid introducing capacity of any kind (particularly long lead wires) into the tube circuit. It is useless to attempt to neutralize the effect of even a small capacity by introducing inductance or resistance. To obtain a pure secondary spectrum, inductance and resistance are to be avoided and at least 0.05 microfarad capacity must be added. With more than $10 \mathrm{ohms}$ resistance or 0.01 millihenry inductance in the tube circuit, a pure secondary can not be obtained by merely adding an excess of capacity.

The names primary and secondary were given by Plücker and Hittorf in their classic monograph on multiple spectra. In recent years the terms have fallen into disuse, but the writer is strongly in favor of their retention. Some specific distinctive names are very desirable. The names band and line spectra are far from being distinctive, while the distinguishing term series spectrum applied by Kayser and Runge to secondary spectra is not sufficiently specific. When more than a single primary spectrum exists (as with nitrogen), it is easy to specify anode primary or cathode primary. And the terms primary and secondary are not likely to be confused with first order and second order applied to grating spectra.

Before discussing the results it may be well to summarize them, together with the results of other workers:

Many elementary gases and vapors possess two entirely different spectra (Plücker and Hittorf). These elements are in number about one-fourth of those known and are largely electro-negative.

The lines of primary spectra do not show the Zeeman effect, while those of secondary do (Runge and Paschen).

The transition from primary to secondary is in most cases very abrupt. Capacity appears to be essential to the production of a pure secondary spectrum. Great current density and a spark gap in series favor, while inductance, resistance, and heated electrodes hinder, the production of secondary spectra. The first inductance added produces the greatest effect per given increment of inductance, while the transition from secondary to primary spectra occurs at about 0.1 millibenry for a great range of capacity or pressure, with or without spark gap in circuit.

Critical capacity is different for different portions of the spectrum, being least in the red and being much greater and rapidly increasing 
at the violet end. It appears to approach a constant value, possibly zero, in the infra red, and the value infinity in the ultra violet perhaps not farther out than $300 \mu \mu$. Critical capacity increases steadily as the density of a gas decreases, becoming suddenly infinite at about one thousandth of an atmosphere.

The electrical conditions concerned with the production of secondary spectra may be stated with some certainty. The steepness of the wave front of the discharge appears to be the principal factor; that is, the rate of variation of the electromagnetic field in the gas or of the number of advancing electrons per unit area of cross section, according to the view taken of the nature of the discharge. Putting in capacity vastly increases the steepness and amplitude of the current wave, while inductance tends to smooth it out again; hence capacity favors the production of secondary spectra, while inductance favors the primary. An external spark gap increases the suddenness of the discharge, while resistance cuts down its amplitude; hence a spark gap helps the production of secondary spectra, while resistance favors the primary. Merely increasing the frequency of a simple alternating (say 2,000 volt) current through a gas should change its spectrum from primary to secondary. But high frequency alone (without amplitude) is not sufficient to produce secondary spectra, for the electrodeless "ring" discharge gives a primary spectrum. In this case the frequency may be as great as $10^{8}$. On the other hand, an intense current (even a continuous one) does tend to change the primary spectrum over to the secondary.

Several theories of the mechanism of radiation have been advanced to account for double spectra. Plücker and Hittorf thought that the secondary spectrum of nitrogen indicated either an allotropic state of the gas or else a chemical decomposition under the violence of the discharge, and were more favorable to the former view. Deslandres, who has done a great deal of work on the primary spectrum of nitrogen, supposes the primary spectrum to be given off by molecules excited by the impact of electrons, while the secondary spectrum is given off by the negative electrons alone. Runge has called attention to the fact that the absence of the Zeeman effect indicates that the primary spectrum comes from uncharged particles or from particles whose masses are large relatively to their charges. Hence, primary spectra might be due to positive ions, secondary to negative ions. But band and line spectra do not exist together, so that it would be necessary to consider the positive electrons taking up all the energy until it became of great intensity and then the negative taking it all. Deslandres has questioned whether positive and negative electrons 
revolving about one another can have other than identical periods. Hemsalech considers the primary nitrogen spectrum due to impacts between positive and negative ions, the secondary to impacts between electrons and positive ions, without, however, discussing a possible mechanism of radiation in either case.

We have to consider a transformation from electrical energy to radiant energy, a change from the energy of convection to energy of wave motion in an extended medium. On the one hand, work on gas conduction has shown beyond question that an electric current in a gas (probably in every medium) consists in a convection of positively and negatively charged particles. The masses, charges carried, numbers and velocities of these charged particles under various conditions, have been determined within narrow limits. On the other hand, Larmor, Lorentz, and others have shown that at least all optical radiation must be due to the motions of charged particles moving with variable velocities. An uncharged particle, if it could exist, would be unable to produce a wave motion in the ether, whatever its motion, while a neutral aggregate of charged particles could lose energy by radiation only when the equilibrium of its internal motion is disturbed by external forces. Here again the magnitude of the charges as well as the acceleration necessary to produce a given radiation has been quite definitely determined.

During the process of disturbance from equilibrium of motion such a neutral aggregate absorbs energy which it radiates in recovering equilibrium. An aggregate not electrically neutral would gradually lose its energy of internal motion until it either broke up and became neutral or until its internal motion ceased. Now the equilibrium of motion of a neutral aggregate could be disturbed by the impact of either an electro-magnetic wave or pulse or of another particle or aggregate. Or the (static) electric field might be so intense as to separate positively and negatively charged particles against their attraction for one another. Undoubtedly all three forms of excitation are effective in producing radiation under various conditions. But disruption appears to occur whenever luminosity is produced, even in the electrodeless "ring" discharge; hence we must always consider the presence of free charged particles and a disruption of part of the neutral aggregate as well as a general disturbance of motion without disruption. And radiation, although not concerned with the steady motion of neutral aggregates, must yet be caused by motions approaching the steady state.

Two forms of atomic structure appear to be capable of steady motion and of radiating observed spectra when this steady motion is 
disturbed, namely, systems in which the satellites (negative electrons) are arranged in rings and those in which the satellites move independently. Under severe stresses a ring system might break down into a system having independent satellites. Considering the ring system as discussed by Thompson as the general type of atom, metallic as well as nonmetallic, we find nearly all the phenomena related to secondary spectra capable of simple interpretation. We should have primary spectra given off by the lateral oscillations of the rings when their steady motion was disturbed, secondary spectra by the independent satellites after the rings had been broken up by violent excitation. The ring systems of the atoms of acid-forming elements are not easily broken up, while those of the metals are already broken up before sufficiently excited to become luminous. The Zeeman effect would be shown only by secondary spectra and the wave lengths of the lines in the primary bands would fall under quite a different spectral series from those of the secondary. Lines of a secondary spectrum would not be related in position to those of the corresponding primary. A nitrogen atom would have as many rings as there are bands in its primary spectrum. Critical capacity would vary with the wave length and become infinite for short waves, because the larger, slower rings would be the first to break up, while the innermost rings could not perhaps be broken up at all. The abrupt and complete transition from primary to secondary spectrum shown by nitrogen, sulphur, iodine, and bromine would indicate that when the discharge exceeds a certain intensity the rings on all the atoms are broken up at once and remain so, while in hydrogen only a portion of the rings would appear to be disrupted at one time, probably on account of very rapid recombination. 


\section{EXPLANATION OF PLATES.}

Plate I. The upper series of spectra are of nitrogren at $1.5 \mathrm{~mm}$ pressure, showing the transition from primary to secondary by gradually adding capacity in steps of $0.03 \mathrm{~m}$. f. The first spectrum of the series (c) is of the white cathode glow. The second spectrum was taken with the same capacity $(0.06 \mathrm{~m}$. f. $)$ as the fifth, but taken in the bulb instead of capillary. The bulb spectrum is seen to be a nearly pure primary, while the capillary is a secondary in the green and yellow, illustrating very well the effect of current density on the production of a secondary. The third spectrum ( $p$ ) is a pure (anode) primary, while those beneath were taken with the capacity indicated. Drawing a line roughly separating primary and secondary, this curve represents critical capacity as a function of wave length.

The lower series shows the same capacity effect in hydrogen. The capacity used is indicated, the bottom spectrum being a nearly pure primary.

Plate II. The upper series shows the same capacity effect in sulphur. The first two spectra are both primary, but the upper of the two was taken when the vapor density was greater.

The lower series of spectra shows the current density effect in hydrogen. The spectrum at the bottom was taken with a very feeble current and long exposure, the second from the bottom with a greater current, the third with the largest current (0.06 amp.) the tube would carry. The two upper spectra were taken with the capacities indicated. $\mathrm{H}_{\beta}$ appears even with a feeble steady current, but $\mathrm{H}_{\gamma}, \mathrm{H}_{\delta}$, and $\mathrm{H}_{\epsilon}$ appear only with heavy current or capacity.

Plate III. This plate shows the effect of inductance in hydrogen and nitrogen in bringing the secondary back to the primary. In each case the secondary at the bottom was taken with a large capacity $(0.1 \mathrm{~m}$. f. $)$. The spectrum just above was taken with the same capacity with a small inductance ( 0.1 millihenry). The next spectrum was taken with the same capacity and a large inductance ( 0.8 millihenry). The topmost spectrum is a pure primary, in each case taken with reither capacity nor inductance. 


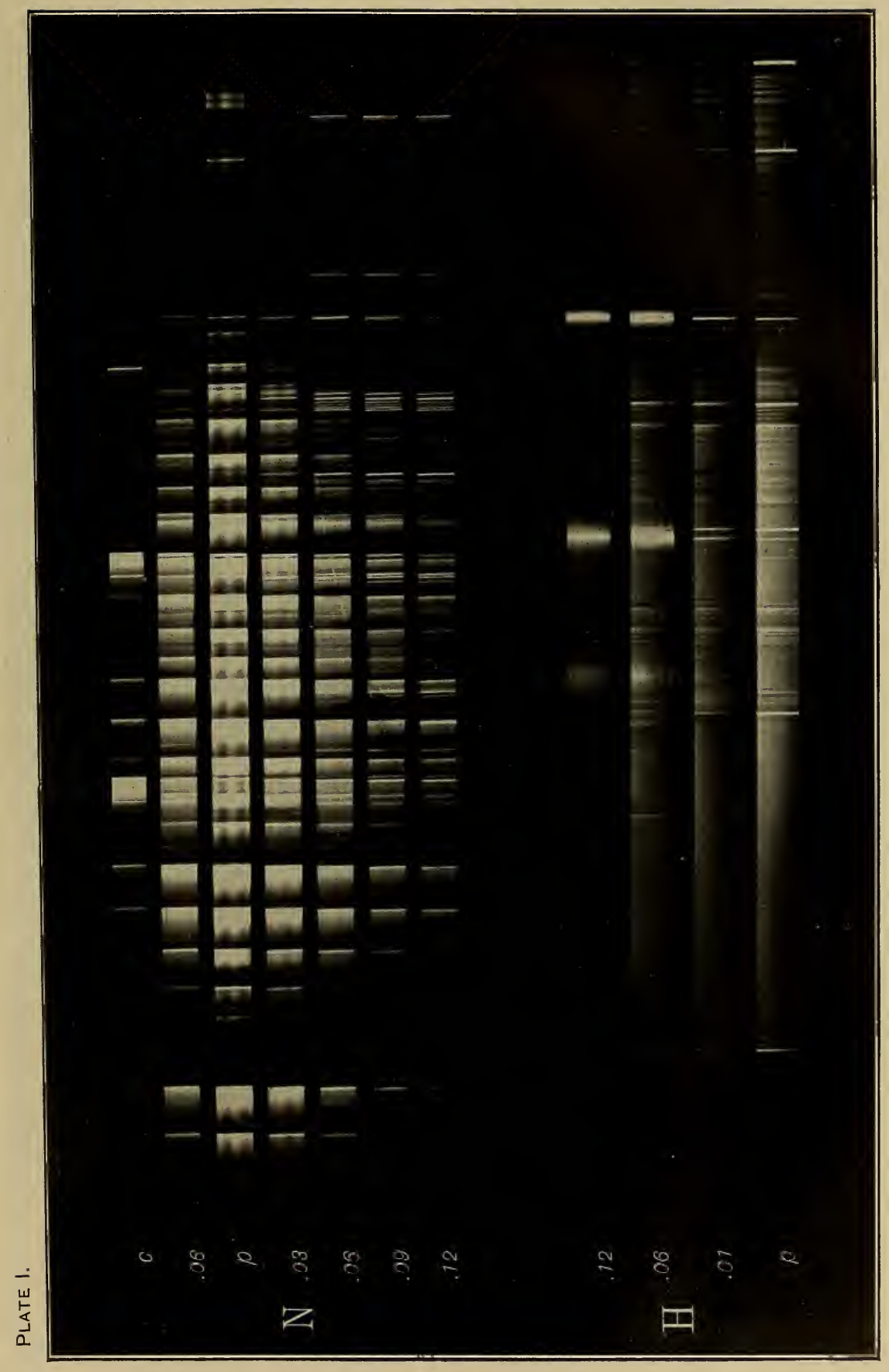




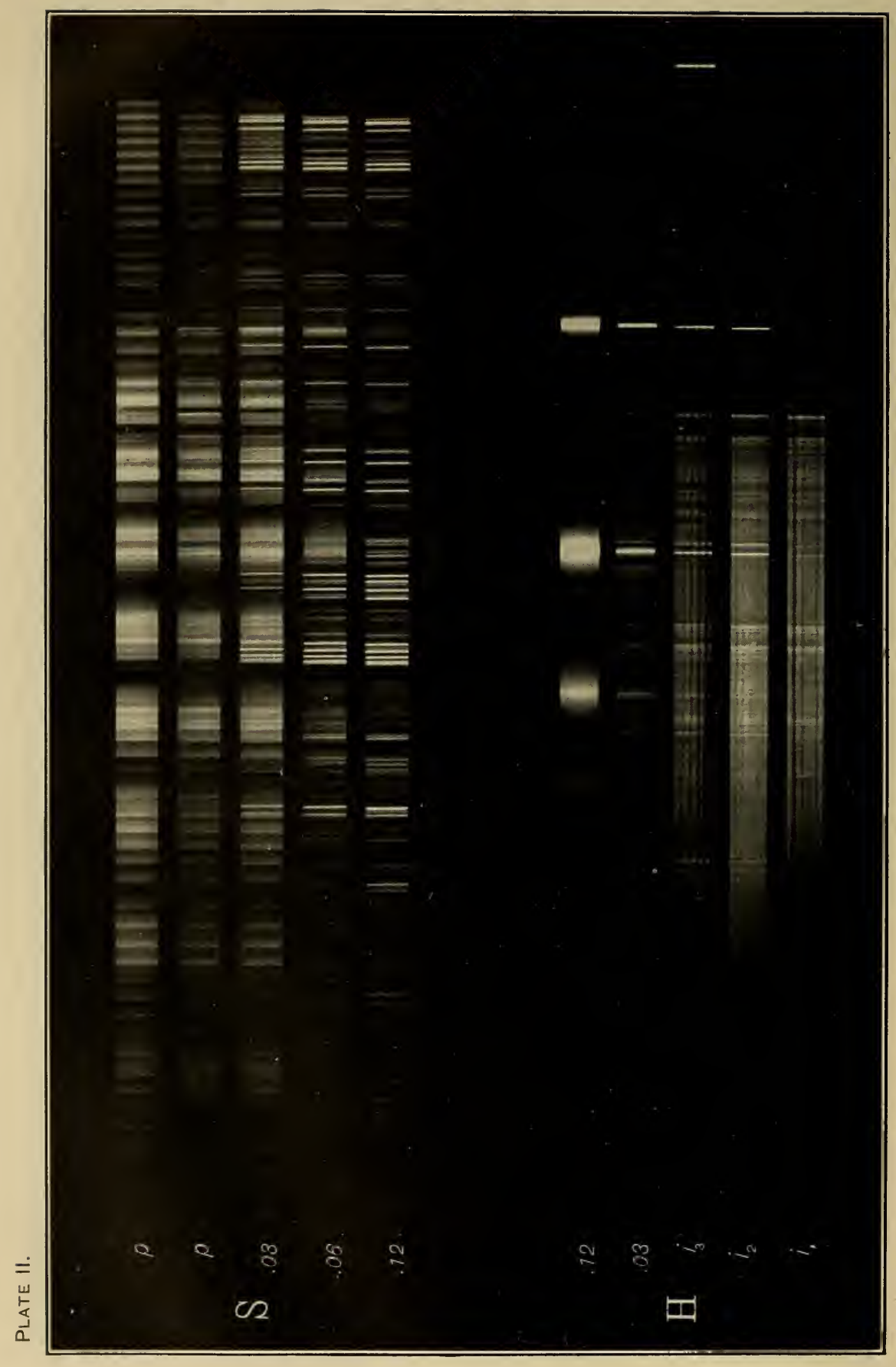




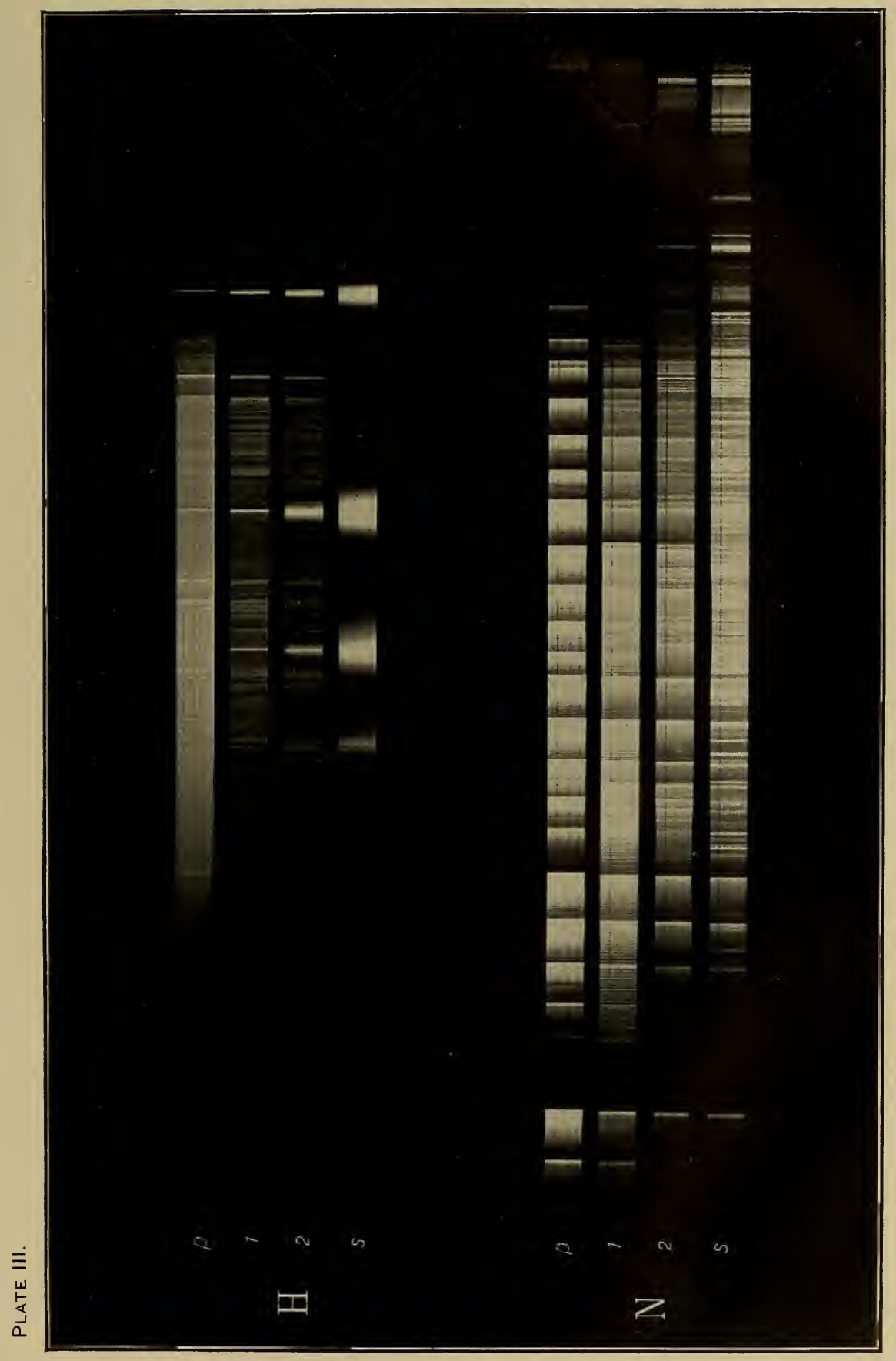




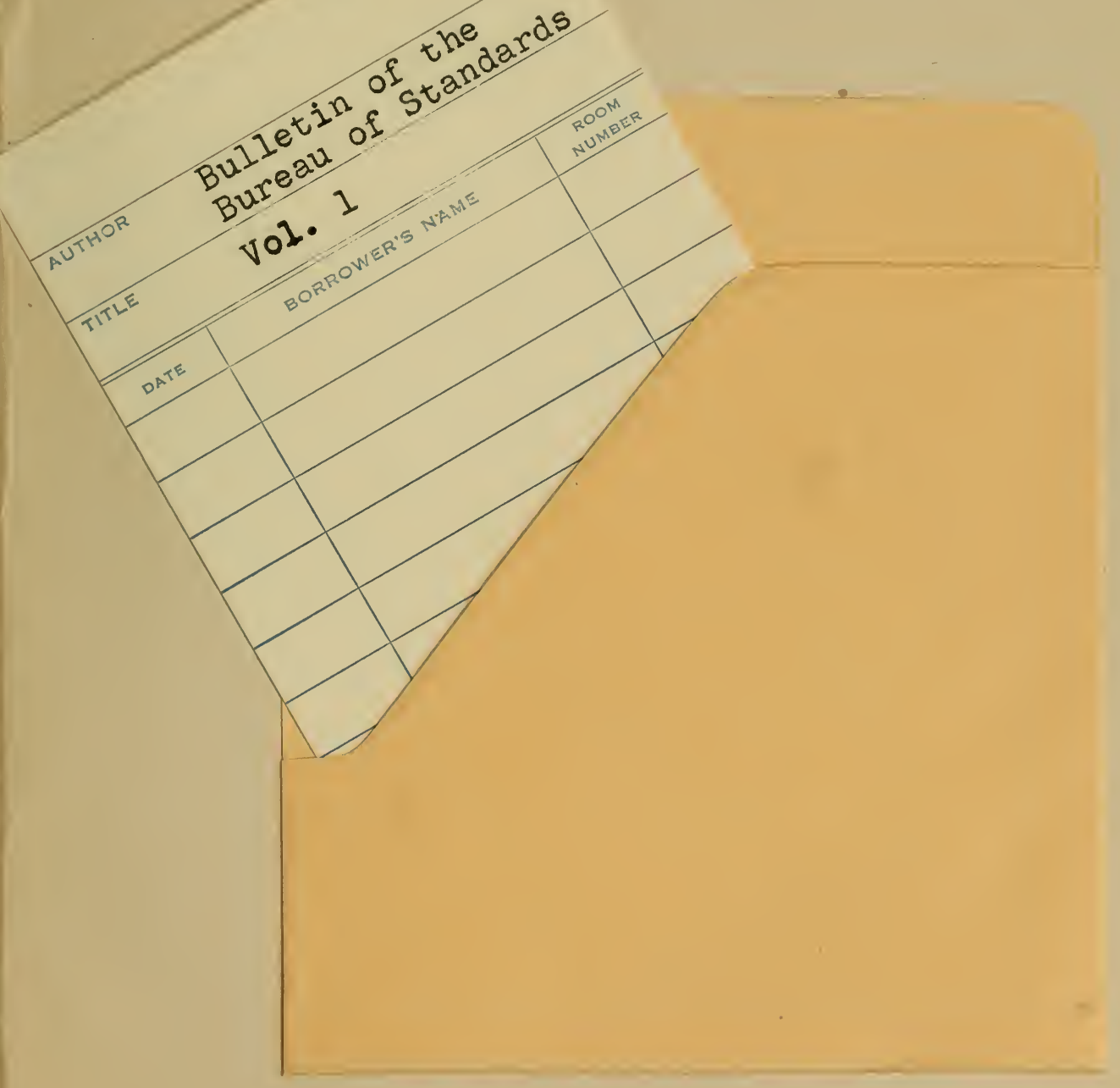


